\title{
VPDC: VIRTUAL PRIVATE DATA CENTER
}

\section{A Flexible and Rapid Workload-Management System}

Mineyoshi Masuda, Yutaka Yoshimura, Toshiaki Tarui, Toru Shonai, and Mamoru Sugie Hitachi, Ltd., Central Research Laboratory

Abstract: Rapid server allocation implemented on Virtual Private Data Center (VPDC), which is an autonomous server allocation system for a three-tier web system, has been developed and tested. The test results show that with this new system elapsed time for application server allocation is about 20 seconds, and that for database server allocation is 140 seconds.

Key words: $\quad$ autonomous, server, allocation, rapid

\section{INTRODUCTION}

Autonomous server-resource management ${ }^{[1][2]}$ is one of the key technologies for current information systems. Server resource management allocates or de-allocates servers in response to fluctuations of system workload. This technology can maintain adequate system capacity; thus, cost performance improves especially in the case of a web transaction system, in which system workload fluctuates frequently.

Adaptive server allocation is one of the key issues concerning easy managements of complex systems. Furthermore, rapid server allocation is another important issue. In particular, rapid server allocation for server-resource management has two advantages:

(1) adaptation to "Slash Dot Effect" (a phenomenon in which a web system receives tens times more accesses than usual in a short time),

(2) improvement in server utilization (because of reduction in extra server resources which act as a "buffer") .

Focusing on a rapid sever allocation, we have developed Virtual Private Data Center (VPDC), an autonomous management system for a three-tier web system. The present study showed that the rapid server allocation implemented on this VPDC system is effective.

The original version of this chapter was revised: The copyright line was incorrect. This has been corrected. The Erratum to this chapter is available at DOI: 10.1007/978-0-387-35674-7_66 


\section{RAPID SERVER ALLOCATION}

System Overview

VPDC is a hosting utility in a data center; it manages several customer systems hosted in a server farm. Figure.1 shows an overview of the VPDC management system. It is composed of a single control server and agents working on each server

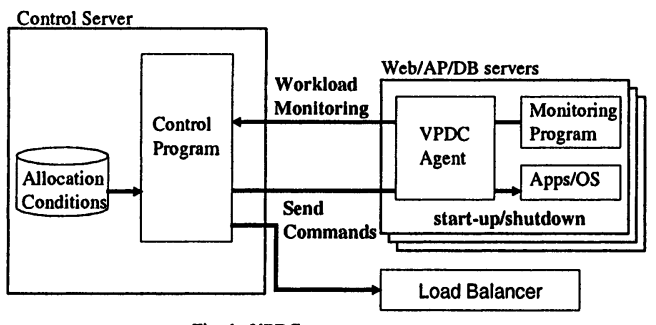

Fig. 1 VPDC management system

(i.e., on both allocated and spare servers). The system has three main functions.

1. Monitoring: The agents on all allocated server periodically obtain load information about a server (i.e., CPU, network, disk utilizations, etc) and the control server gathers them.

2. Decision making: The control server analyzes load information in accordance with a predefined allocation policy for each customer and makes server allocation/deallocation decisions.

3. Action: The agent receives a set of commands for start/stop application, and then the control server changes the configuration of a load balance group.

\section{Implementation}

For rapid server allocation, the following two approaches are implemented on the VPDC.

(1) Cutting out data copy

Figure. 2 shows typical and VPDC server allocation sequences (the length of each rectangle means a time cost). In the first and the third parts in the typical server allocation [shown in Fig.2 (a)], data copy to a local server takes a long time (several minutes). In VPDC server allocation, such data copying is cut out by the following methods.

- OS installation is not executed during a server allocation time. OS is preinstalled, and for the purpose of security maintenance, the OS is initialized during a server deallocation time.

Shared file server is used for deploying application programs.

The VPDC server allocation parts are shown in Figure.2 (b). The total server allocation time is considerably shortened and the application program start-up time dominates.

(2) Eager allocation and lazy deallocation

The control server makes the decision to allocate servers as soon as it detects the signs of a slash-dot effect. This policy might make mistakes if, for example, the workload returns to a normal level in a short period. However, this policy is adapted, since VPDC gives preference to avoiding late server allocation in the case of the slash-dot effect. In contrast, to prevent repeated server allocation and deallocation in a ping-pong fashion, a server deallocation decision is made only after the workload has stayed low for a certain time. 


\section{EVALUATION}

\section{Prototype System}

Figure. 3 shows the construction of the VPDC prototype. The NFS server provides application programs and contents data. The VPDC agent software has been developed as a SNMP agent to which VPDCspecific MIB modules are installed. Agents run on all servers including spare servers. Apache runs on the web servers, Tomcat with a hand-maid session handover runs on the AP servers, and Oracle Parallel Server (OPS) runs on the DB servers.

\section{Results}

Experiments on the VPDC prototype are performed to confirm the effectiveness of VPDC rapid server allocation. The test program running on the prototype is a subset of TPC-W $\mathrm{W}^{[3]}$. System workload is increased in order to load the DB and AP servers so that server allocations are triggered.

Figure. 4 shows relative amount of accesses to the prototype, average response time measured on a client, CPU utilization of allocated servers, and elapsed time for server allocation per layer. Each vertical line means an event time such as a server allocation. These results show that elapsed time for the AP server allocation is about 20 seconds. This is because the control server takes 10-15 seconds to detect the sign of the slash-dot effect. On the other hand, the time for the DB server allocation is about 140 seconds. This is because DB application initialization takes a long period before the DB server is ready to receive requests. After the DB-layer bottleneck is removed, the AP-layer is able to send a larger amount of requests to the DB-layer. Thus, the control server allocates server AP\#2.

\section{CONCLUSION}

A rapid server allocation implemented on VPDC was developed and evaluated. Due to server allocation without a disk copy, in the application layer, VPDC improves the site's response time to within approximately 20 seconds after the start of the slash-dot effect. Workload management of the DB layer improves the site's response time to within 140 seconds. Elapsed time for the AP server is rapid enough, but that of the DB server is rather slow because of the start-up time of the DB application. 


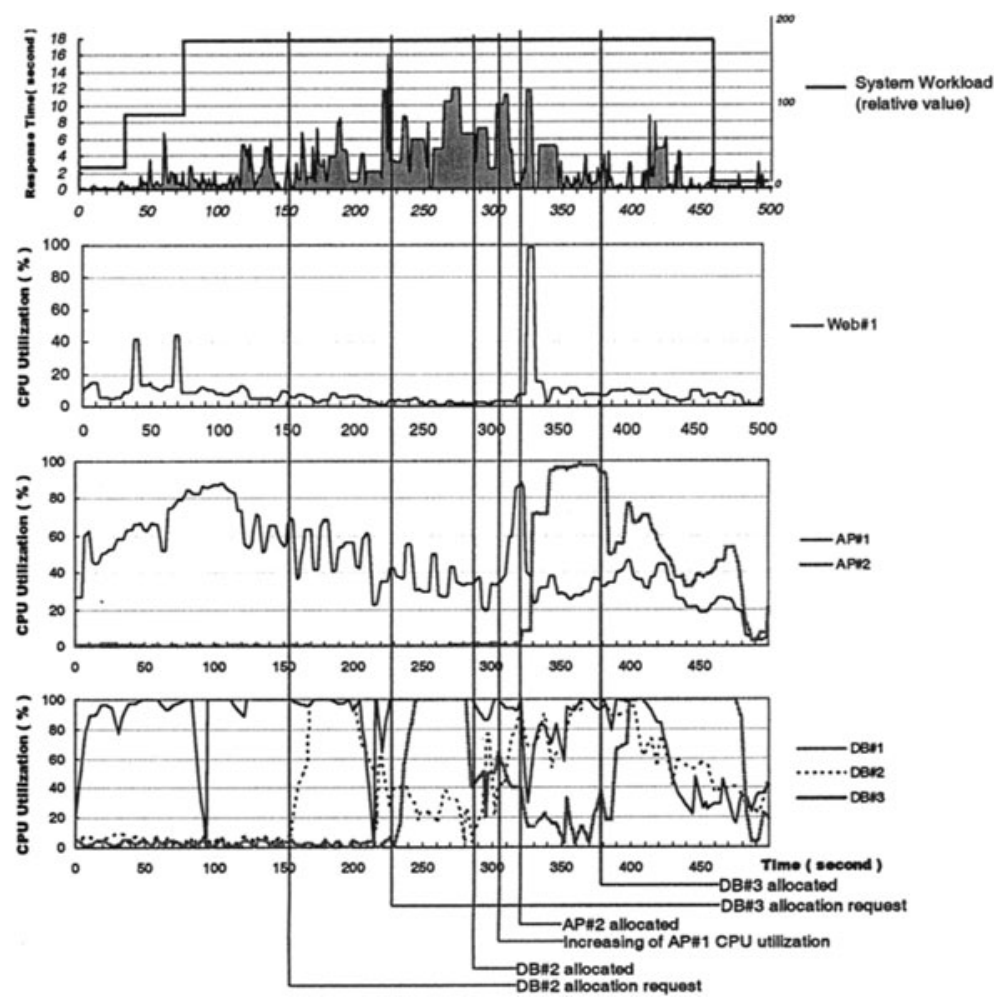

Fig. 4 Relationship between workload, response time, and server utilization

\section{ACKNOWLEDGEMENTS}

We would like to thank all the people who supported the VPDC development.

\section{REFERENCES}

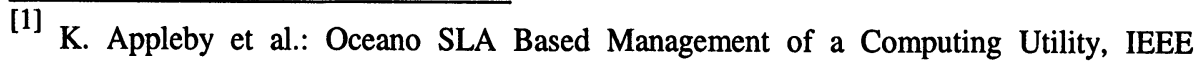

[2] International Symposium on Integrated Network Management 2001, (2001)

[2] L.L. Fong et al.: Dynamic Resource Management in an eUtility, NOMS2002 (2002)

[3] “TPC-W" http://www.tpc.org/tpcw/default.asp 